\title{
Reporter Composite Map
}

National Cancer Institute

\section{Source}

National Cancer Institute. Reporter Composite Map. NCI Thesaurus. Code C44260.

A ReporterCompositeMap is the description of how source Reporters are transformed into a target CompositeSequences. For instance, several reporters that tile across a section of a chromosome could be mapped to a CompositeSequence. (caMAGE) 